\title{
Factores de estancia hospitalaria prolongada en una in- stitución de salud de Medellín
}

\author{
Factors of extended hospital stay in a health care institution in Medellin \\ Fatores de estância hospitalar prolongada numa instituição de saúde de Medellín
}

\author{
Natalia Cañas-Benítez ${ }^{1}$ \\ Erika Moreno-Pareja ${ }^{2}$ \\ Edwin Alexis Cataño-Saldarriaga ${ }^{3}$
}

\section{Resumen}

Objetivos: el objetivo de este proyecto de investigación se basó en identificar los factores asociados a la estancia hospitalaria prolongada, en una institución de mediana y alta complejidad de Medellín. Materiales y métodos: se realizó un estudio analítico, cuantitativo, de corte transversal, con una selección aleatoria simple de pacientes registrados en el consolidado de estancia hospitalaria prolongada en la institución objeto de estudio, donde $(n=291)$ pacientes. Para el análisis bivariado se utilizó x2 cuadrado en SPSS, los valores P arrojados fueron evaluados con un nivel de confianza del 95\% y un margen de error del 5\%, con un nivel de significancia o de valor alfa de 0.05 de los 291 registros analizados. Resultados: se encontró asociación estadísticamente significativa entre estancia hospitalaria prolongada y atención domiciliaria $(\mathrm{p}=0,028)$, con procedimientos $(\mathrm{p}=0.046)$ y ayudas diagnósticas $(\mathrm{p}=0,38)$. Conclusiones: La estancia hospitalaria de un paciente, no solo depende de su condición clínica, sino de una interrelación con otros actores en su atención como la institución, el prestador y la red de servicios.

Palabras clave: Hospitalización, procedimiento, diagnóstico, atención domiciliaria.

\section{Abstract}

Objectives: The objective of this research project was to identify associated factors of extended hospital stay, in a medium and high-complexity health care institution in Medellin. Materials and methods: An analytic, quantitative, cross-sectional study was made, with a simple random sample of patients $(n=291)$ from the consolidate records of extended hospital stay in the institutions subject of study. For the bivariate analysis, the x 2 squared was used in SPSS, the P values generated were evaluated with a confidence level of $95 \%$ and a margin of error of $5 \%$, with a significant value or alpha value of 0.05 from the 291 records analyzed. Results: A significant statistical association was found between prolonged hospital stay and home care $(p=0,028)$, with procedures $(p=0.046)$ and diagnostic aids $(p=0.38)$. Conclusions: The hospital stay of a patient, does not only depend on their clinical condition, but also on the correlation with other care actors such as the institution, provider, and service network.

Key words: Hospitalization, procedure, diagnosis, home care.

\section{Autor de correspondencia*}

${ }^{1 *}$ Enfermera. Magister en administración en salud. Clínica Medellín, Colombia. Correo: naty.c26@hotmail. com, (D) 0000-0001-7947-9883

${ }^{2}$ Enfermera. Magister en administración en salud. Metrosalud Medellín, Colombia, Correo: morenoparejaerika@gmail. com, (iD 0000-0001-5269-0347

${ }^{3}$ Enfermero. Magister en calidad en salud. Profesor de catedra. Universidad de Antioquia, Facultad de enfermería. Medellín, Colombia. Correo: Edwinenfer2010@gmail.com (D) 0000-00031941-8549

Recibido: 27 diciembre 2020

Aprobado: 16 abril 2021

Para citar este artículo / To reference this article / Para citar este artigo:

Cañas-Benítez N, Moreno-Pareja E, Cataño Saldarriaga EA. Factores de estancia hospitalaria prolongada en una institución de salud de Medellín. Rev. cienc. cuidad. 2021; 18(2):43-54. https://doi. org/10.22463/17949831.2821

(C) Universidad Francisco de Paula Santander. Este es un artículo bajo la licencia CC-BY-NC-ND 


\section{Scientific Journal of Nursing}

\section{Resumo}

Objetivo: Identificar os fatores associados à estância hospitalar prologada numa instituição de mediana e alta complexidade de Medellín. Materiais e métodos: Estudo analítico, quantitativo, transversal, com uma seleção aleatória simples de 291 pacientes registrados no consolidado de estância hospitalar prolongada numa instituição de saúde. Para a análise bivariada usou-se o X 2 em SPSS, os valores p obtidos foram avaliados com um nível de confiança do 95\% e uma margem de erro do 5\%, considerando significativos de alfa de 0,05. Resultados: Encontrou-se associação significativa entre a estância hospitalar prolongada e a assistência domiciliar $(\mathrm{p}=0,028)$, com procedimentos $(\mathrm{p}=0,046)$ e ajudas diagnosticas $(\mathrm{p}=0,038)$. Conclusões: A estância hospitalar de um paciente não só depende da sua condição clínica, e sim da interação com outros atores no seu atendimento como a instituição, o prestador e rede de serviços.

Palavras-chave: Internação, procedimento, diagnostico, assistência domiciliar.

\section{Introducción}

En los países latinoamericanos, la desproporción entre oferta y demanda de servicios de salud es motivo de preocupación. En el caso de Colombia, el sistema de salud enfrenta una crisis por la incontenible demanda de los servicios de salud, que supera la capacidad de los hospitales para atender pacientes. Situación que se ve reflejada en la saturación de los servicios de urgencias, hasta tal punto, en que se cierran temporalmente. Teniendo en cuenta que los servicios de urgencia son la principal puerta de entrada a los servicios de hospitalización, que corresponden al $70-75 \%$ de los ingresos hospitalarios, es común que cuando los pacientes llegan a un hospital no haya disponibilidad de cama para atender su necesidad (1).

Cabe señalar que la estancia hospitalaria prolongada constituye una preocupación y por tanto se convierte en objeto de estudio, sobre todo cuando se trata de una institución de mediana y alta complejidad en la ciudad de Medellín; esta inquietud también se ve reflejada a nivel nacional, ya que genera efectos negativos en el sistema de salud como: aumento en los costos, deficiente accesibilidad a los servicios de hospitalización, saturación de las urgencias y riesgo de eventos adversos (1). La estancia hospitalaria prolongada es un indicador indirecto de la calidad del cuidado otorgado a los pacientes en las unidades de segundo y de tercer nivel, ya que un procedimiento retrasado, omitido o excesivo prolongará la estancia hospitalaria de los pacientes, incrementando los costos (2). Los costes asociados a la hospitalización han aumentado de manera exponencial. Es por eso, que la reducción de la estancia hospitalaria se ha planteado como estrategia para optimizar los re- cursos sanitarios (3).

Desde esta perspectiva, un servicio de hospitalización con estancias prolongadas de pacientes puede considerarse como indicador de falta de eficiencia en el flujo de pacientes. Además de la relación directa entre la prolongación de la estancia y la disponibilidad de camas, también aumentan los costos de la atención, el riesgo de eventos adversos y la mortalidad (4).

Por otra parte, la dificultad en este contexto se intensifica por la demanda creciente para tener acceso a servicios de salud, en circunstancias en que existe una presión aún más fuerte sobre los gobiernos de América Latina, para reducir los gastos del sector público, con el propósito de estabilizar las economías de estos países (5). La estancia hospitalaria prolongada se encuentra asociada con: es los riesgos de infección, eventos adversos, fatiga del cuidador, trastornos psicológicos en el paciente, solvencia del sistema de salud y eficiencia de las instituciones, por lo que una buena gestión redundará en una mejor calidad del sistema de salud (6). Así mismo, las instituciones prestadoras de servicios de salud reciben miles de usuarios, los cuales se han visto afectados, ya que muchos de sus requerimientos no siempre se resuelven convenientemente, lo que aumenta la ineficiencia en los servicios de salud, situación que también viven las instituciones de mediana y alta complejidad en la ciudad de Medellín, en particular la que es objeto de este estudio, donde se producen estancias hospitalarias prolongadas.

La institución objeto de este estudio presta servicios de salud de mediana y alta complejidad; se encuentra ubicada en el área metropolitana; cuenta con 2 sedes 


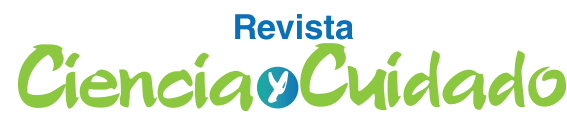

Scientific Journal of Nursing

situadas en la zona sur y occidental de Medellín. En esta institución se evidenció un aumento en el registro de días de estancia prolongada, dando como resultado el que se afecte el indicador de giro cama. Al respecto, el número de pacientes que pasaron por cada cama disponible de la institución se redujo, debido a un promedio de 38 altas diarias para el 2018, con respecto al año de estudio con un promedio de 25 altas diarias; obteniendo como resultado el colapso en el servicio de urgencias e insatisfacción del cliente interno y externo. Estos factores afectan de manera negativa la eficiencia de la institución. Se precisa que los datos anteriores se obtuvieron de la institución objeto de estudio.

Hay que mencionar además, que durante los últimos veinte años, América Latina ha experimentado un proceso gradual de transformación institucional en los sectores de la educación, la atención de la salud y la seguridad social. Las reformas en el sector de la salud han perseguido los mismos objetivos que en otras regiones del mundo, esto es, aumentar la eficiencia, al tiempo que se enfrenta a un acentuado crecimiento de la demanda y de los costos (7).

Se debe agregar que el uso racional y la optimización de los recursos se reflejan positivamente en los indicadores de calidad de una institución hospitalaria; igualmente, el incremento de la calidad técnico científica del personal de salud aumenta, así como el uso eficiente de los recursos, lo que reduce los eventos adversos (8).

Lo anterior evidencia la importancia del análisis de los factores asociados con la estancia hospitalaria prolongada en esta institución de salud. De ahí, que sea importante estimular la adquisición de nuevos conocimientos, mediante la realización de un estudio minucioso. De igual forma hay que abordar el tema de los factores asociados a la estancia hospitalaria prolongada y entregar datos que puedan fortalecer la gestión del recurso físico de la entidad, teniendo en cuenta además que en la actualidad los servicios de hospitalización soportan una demanda insatisfecha por la alta ocupación.

El propósito de esta investigación es brindar información, a través de los resultados, a los directivos de la Institución como herramienta verídica, con datos actualizados, frente al deterioro de algunos indicadores hospitalarios, de tal forma que permitan facilitar la toma de decisión gerencial o administrativa, una vez identificados los factores asociados a la estancia prolongada, que se reflejará en la disminución de costos. La presente investigación se considera relevante debido a que proporcionará beneficios a la organización, ya que se busca suplir el desconocimiento que se tiene acerca de la
Natalia Cañas Benítez, Erika Moreno Pareja, Edwin Alexis Cataño Saldarriaga.

estancia hospitalaria prolongada. A tal fin se valoraron factores tanto administrativos como asistenciales, que sirvieron para analizar este fenómeno y poder intervenir asertivamente en planes y acciones enfocadas a su control, influyendo en la evaluación sobre los costos de la institución, en una mayor oportunidad y calidad en la atención, de tal forma que se genere reconocimiento por parte de los usuarios y aseguradoras.

\section{Objetivo}

Identificar los factores asociados a la estancia hospitalaria prolongada en una institución de mediana y alta complejidad de Medellín.

\section{Materiales y Métodos}

El enfoque metodológico de esta investigación fue de carácter cuantitativo y con intención analítica; es decir, los resultados se expresaron mediante magnitudes numéricas, con el fin de facilitar el análisis estadístico. En este estudio, el análisis de los datos permitió obtener información que dio cuenta de los diferentes procesos y posibles relaciones con la estancia hospitalaria prolongada. El periodo de recolección de los datos se realizó desde el 1 de enero de 2018 hasta el 31 de diciembre del mismo año. Se analizaron las historias clínicas completas de aquellos pacientes que fueron hospitalizados durante el período mencionado. El método empleado fue analítico, en donde la labor del investigador se limitó a la medición de las variables que se tuvieron en cuenta en el estudio. Se buscó identificar cuáles fueron los posibles factores asociados a la estancia hospitalaria prolongada en una institución de mediana y alta complejidad en la ciudad de Medellín (9). Además, es un estudio transversal porque se examinaron las variables en un tiempo determinado, se recolectaron datos en un período único, en un solo momento, para medir la incidencia de una exposición y/o resultado en una población definida y en un punto específico, no involucró seguimiento. En este caso se determinó con la presencia de la estancia hospitalaria prolongada $(6,10)$.

Como se dijo antes, la unidad de análisis de este estudio fueron las historias clínicas de los pacientes hospitalizados en una Institución Prestadora de Servicios de Salud de mediana y alta complejidad, identificadas con la estancia hospitalaria prolongada.

Hay que mencionar, además, que la población de referencia la conformaron los pacientes que ingresaron al 


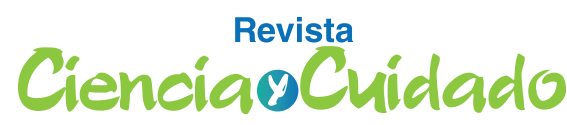

Scientific Journal of Nursing

servicio de hospitalización de una institución de mediana y alta complejidad en la ciudad de Medellín. El total de registros del consolidado de estancia hospitalaria prolongada fue de 1921 historias clínicas en el año 2018, de las cuales se excluyeron 720 historias pertenecientes a otras sedes de atención hospitalaria, para un total de 1201 restantes. Con estas historias se realizó un muestreo aleatorio simple, con un nivel de confianza de $95 \%$, un margen de error del $5 \%$ y una proporción esperada del $20 \%$; por tanto, la muestra calculada fue de 291 registros, los cuales fueron analizados.

Los pacientes que fueron excluidos de la investigación, son aquellos:

- Cuyas historias clínicas están incompletas.

- Los que son atendidos en sedes hospitalarias diferentes a la institución objeto de estudio.

Conviene aclarar, que para este estudio se aplicó el control de sesgos de selección, debido a que la información analizada procedía de una base de datos, que pudo verse afectada en cuanto al tiempo de su recolección, ya que durante los fines de semana no se realizaban los respectivos registros.

Por otro lado, para el control de los sesgos de confusión se analizaron aquellas variables que pudieran modificar el comportamiento del estudio, con el análisis bivariado.

La información recolectada se digitó en Microsoft Excel 2013; posteriormente se procesó en el programa SPSS versión 18,0 .
Además, las variables cuantitativas se presentaron en tablas con porcentajes y se realizaron medidas de tendencia central con la variable edad; también, se utilizaron gráficos de barras.

Con respecto, a las variables cualitativas se realizó el x2 cuadrado en SPSS; se debe agregar que los valores $\mathrm{P}$ arrojados por el estadístico fueron evaluados con un nivel de confianza del $95 \%$, lo que significa que el valor alfa tomado fue de 0.05 .

En definitiva, el presente estudio se considera sin riesgo, porque es cualitativo, de tipo descriptivo exploratorio, en donde no hubo manipulación de variables; de igual forma, la fuente de información fue una base de datos prediseñada, con la aprobación del Comité de Ética institucional, mediante el acta N. 01-2017.

\section{Resultados}

Se analizaron 291 registros de pacientes con estancia hospitalaria prolongada, en una institución de mediana y alta complejidad en la ciudad de Medellín. Los resultados obtenidos en el presente estudio son los siguientes:

\section{Características sociodemográficas de la población con estancia hospitalaria prolongada}

Del total de registros, el $49.83 \%$ (145) fueron mujeres y el $50.17 \%$ (146) hombres. Se logra identificar que, en la distribución porcentual por sexo, no hay una variación estadísticamente significa entre ambos (Ver Figura 1).

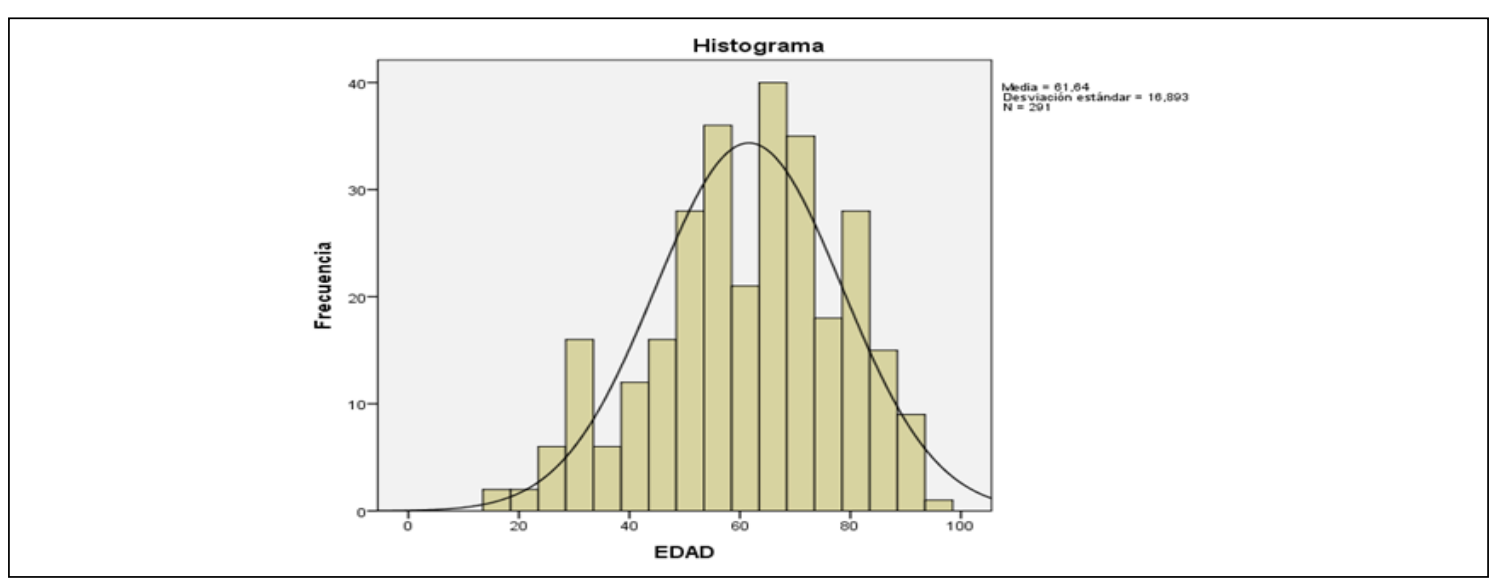

\begin{tabular}{|c|c|c|c|c|c|c|c|c|}
\hline Variable & $\mathrm{N}$ & Media & Mediana & Moda & Promedio & Minimo & Maximo & DE \\
\hline Edad & 291 & 55,4 & 64 & 58 & 61 & 16 & 94 & 16,86 \\
\hline
\end{tabular}

Figura 1. Medidas de frecuencia central y dispersión de la edad de los pacientes, en una IPS de mediana y alta complejidad en Medellín.

Fuente: Autores del proyecto 


\section{Scientific Journal of Nursing}

Del total de registros analizados se logró determinar que la edad promedio fue de 61 años, con una desviación estándar de 16,8 años y una mediana de 64 años, identificando que el $33 \%$ de los pacientes se encontraban en un rango de edad de 61 a 75 años (Ver Figura 2).

Desde otro punto de vista, el $27 \%$ de los registros cor- responde al rango de edad de 46 a 60 años, el 22\% a pacientes con 76 y más años; de igual manera el 11\% de los pacientes se encuentra en un rango de edad de 31 a 45 años. No obstante, la población con menor porcentaje son los pacientes con edades que oscilan en el rango de 16 a 30 años con un 7\% (Ver Figura 2).

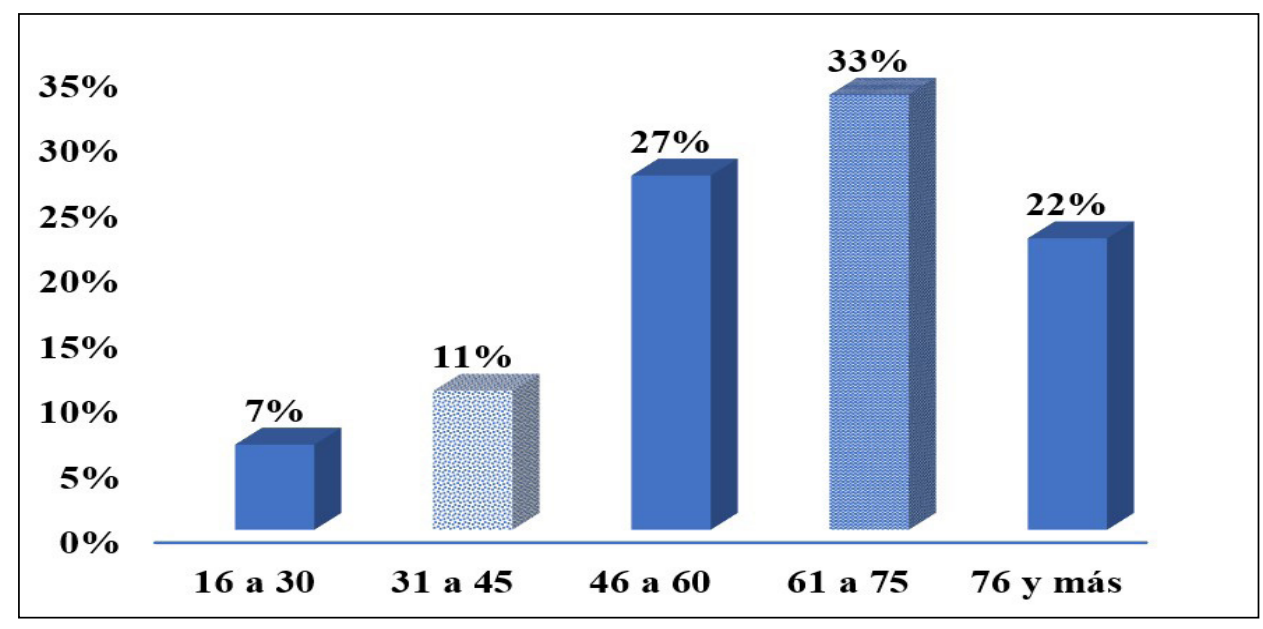

Figura 2: Distribución porcentual de los pacientes con estancia prolongada, por rango de edad, en una IPS de mediana y alta complejidad en Medellín.

Fuente: Autores del proyecto

Con relación, al estrato socioeconómico de los pacientes que se analizaron se encontró que el 55\% (159) pertenecen al estrato 3; en este grupo se encuentra la mayoría de los usuarios registrados en la base de datos. Además, el 16\% (46) corresponde al estrato 2, el 14\% (41) al estrato 4, el 11\% (32) están encuestados en el estrato 1 y por último el $4 \%$ (13) al estrato 5 .

Del total de los registros analizados, se detecta que la mayoría de los usuarios pertenecen al régimen contributivo, con el 77\% (224), seguidamente hay un $12 \%$ de usuarios afiliados al régimen subsidiado (34), el 10\% pacientes de pólizas y particulares (28) y un $2 \%$ (5) con convenios especiales.

Por otra parte, en los registros analizados se observa que el $72 \%$ (210) corresponden a las EPS: Coomeva,
Sura y Savia Salud. Además, se encontró que el 74,91\% fueron pacientes no quirúrgicos frente al $25 \%$ que son pacientes quirúrgicos. La mayor población se concentra en pacientes de medicina interna y otras especialidades no quirúrgicas.

Factores individuales e institucionales asociados a la estancia hospitalaria prolongada.

De acuerdo a lo observado y según los resultados, se ve que los factores que más influyen son de origen institucional. En los registros analizados se encontró que el 77\% (224) corresponde a factores institucionales, el $22 \%$ (64) a factores sociodemográficos y el 1\% (3) a factores individuales. 


\section{Scientific Journal of Nursing}

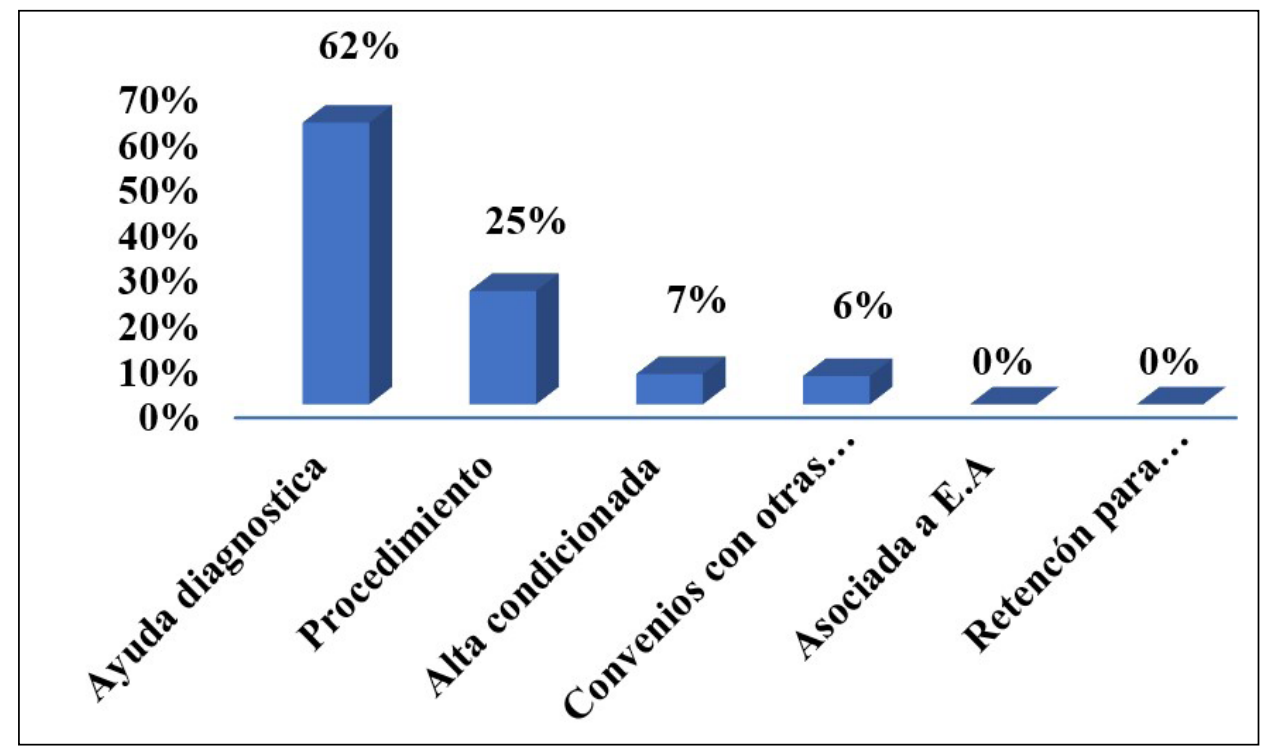

Figura 3: Distribución porcentual por factores institucionales, de pacientes con estancia prolongada, en una IPS de mediana y alta complejidad de Medellín.

Fuente: Autores del proyecto

De los registros analizados correspondientes a factores institucionales, se identificó que el $62 \%$ de estancias hospitalarias prolongadas pertenecen a ayudas diagnósticas y el $25 \%$ a procedimientos, lo que indica que la demanda superó la oferta, para el año objeto de estudio (Ver Figura 3).

Además, de los registros de pacientes con estancia hospitalaria prolongada se encontró que para el 62\% (179) su permanencia es justificada en la institución, si se tiene en cuenta que los requerimientos ameritaban estar en la IPS objeto de estudio, sin que pudiesen salir hasta resolver su necesidad. Para el $38 \%$ no se consideró pertinente su estancia debido a que la resolución de las necesidades de los pacientes se podían resolver en IPS de menor nivel de complejidad e incluso en el domicilio. Así mismo, de los registros analizados se observó que respecto a los factores individuales relacionados con los recursos económicos, el 1\% (3) de los pacientes presentaron estancia hospitalaria prolongada, debido a la falta de recursos económicos, demostrándose la dificultad para el regreso al domicilio una vez dado de alta. También se observó que no se presentaron casos de estancia hospitalaria prolongada relacionados con falta del apoyo familiar (Ver Figura 3).

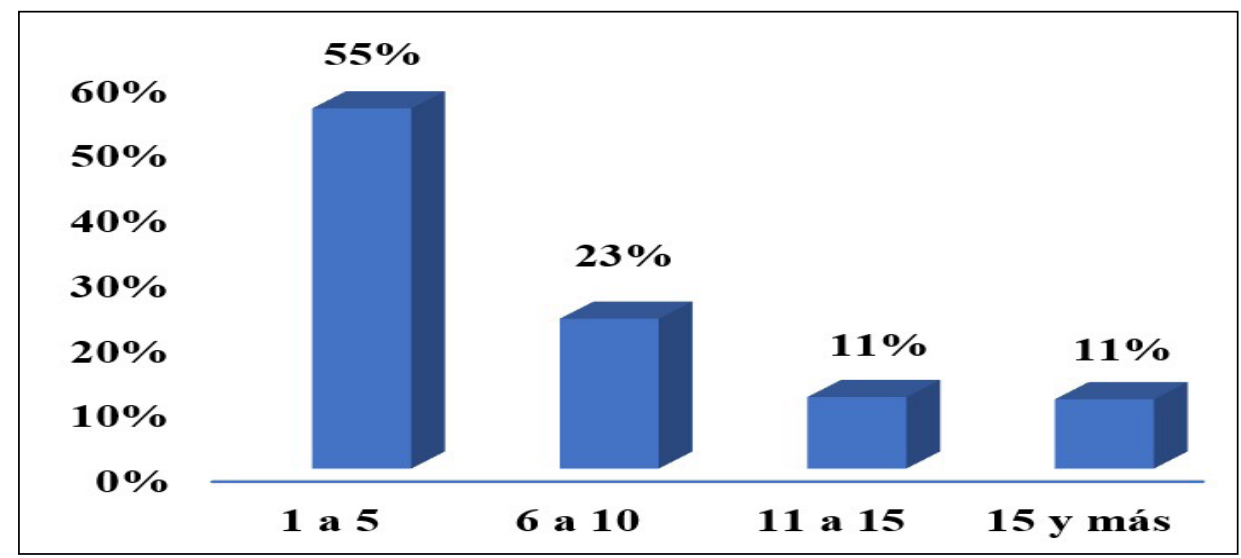

Figura 4: Distribución porcentual por rango de días de estancia hospitalaria prolongada, en una IPS de mediana y alta complejidad de Medellín.

Fuente: Autores del proyecto 


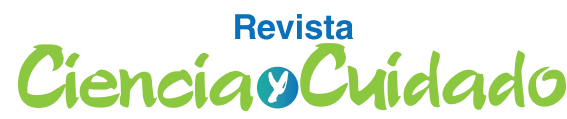

Scientific Journal of Nursing

Así mismo, del total de los registros analizados se concluyó que el 22\% (64) presentaron dificultades en el acceso y cobertura para la atención domiciliaria, debido a la georreferencia que realizan las dispensadoras de medicamentos domiciliarios y aseguradoras.

Por otro lado, de los registros analizados se encontró que el 55\% (161) de pacientes tuvieron una estancia en el rango de 1 a 5 días, si se tiene en cuenta que estos son días de estancia hospitalaria no justificada dentro del total de la hospitalización. El 23\% (67) de pacientes en el rango de 6 a 10 días, el 11\% (32) y 11\% (31) en los rangos 11 a 15 o más días, respectivamente (Ver Figura 4).

Del mismo modo, en los registros analizados se evidencia que en el rango 1 el 50,3\% fueron hombres; en el rango 2 predominaron las mujeres con un 50,7\%; en el rango 3 se encuentran los hombres con un 56,3\%, y en el último rango están las mujeres con un $54,8 \%$. Respecto a ambos sexos no se obtuvo mayor diferencia (Ver Figura 4).

Según el análisis de los registros además se dedujo que en todos los rangos relacionados con los días de estancia, predominó el régimen contributivo con un porcentaje total del $77 \%$ que corresponde a 224 pacientes. Como dato relevante para el rango 4 de 16 y más días se observó que el $22 \%$ pertenecen al régimen subsidiado. Igualmente, se determinó que la especialidad que tiene mayor estancia hospitalaria prolongada fue la no quirúrgica, con un total de $74,9 \%$, equivalente a 218 registros, especialmente en las áreas de medicina interna, neurología, cardiología y neumología. El rango de 16 y más días presentó el $80,6 \%$ de pacientes no quirúrgicos, con estancia hospitalaria prolongada.

Con respecto, al análisis de los registros por rango de días de estancia hospitalaria prolongada en función del estrato socioeconómico, se determinó que los pacientes que presentaron mayor registro en todos los rangos fue el estrato 3 con un total de $54,6 \%$ correspondiente a 159 , pertenecientes en su mayoría al régimen contributivo. Al tener en cuenta la edad, se dedujo que los pacientes pertenecientes al estrato 5 fueron quienes presentaron mayor estadía con un porcentaje del 33,3\%, debido a que en este rango se concentra la población del adulto mayor, quienes demandan más servicios hospitalarios. Del total de los registros analizados se concluyó que los factores que causaron mayor estancia hospitalaria fueron los institucionales, con una proporción total de $77 \%$ equivalente a 224 pacientes, con una significancia estadística respecto a los demás factores.

En cuanto al análisis del total de los registros con respecto a las causas de estancias prolongadas institucion-
Natalia Cañas Benítez, Erika Moreno Pareja, Edwin Alexis Cataño Saldarriaga.

ales se determinó que la mayor significancia estadística, corresponde a las ayudas diagnosticadas con una proporción de 47,8\% (139) pacientes. Sin embargo, para el año 2018, la institución no contaba en su portafolio de servicios con ayudas diagnósticas requeridas para las demandas solicitadas por los pacientes.

Desde otro punto de vista, de los registros analizados se comprobó que el 22\% (64) presentaron dificultades en el acceso y cobertura para la atención domiciliaria. En esta situación, se encontró un promedio de 13 pacientes, quienes permanecieron más días de estancia, en una proporción de $41,9 \%$ para el rango 4; también muchos pacientes permanecieron hospitalizados esperando oxígeno y medicinas domiciliarias, para la continuidad del tratamiento extra hospitalario.

Otro aspecto hace referencia al análisis de los registros por rango de días de estancia, comprobándose que el $1 \%$ (3) experimentaron una estancia hospitalaria prolongada por falta de recursos económicos. Con base en los datos anteriores, se encontraron asociaciones estadísticamente significativas entre estancia hospitalaria prolongada y atención domiciliaria, procedimiento y ayudas diagnósticas con un valor $p=0,028, p=0,046$ y $\mathrm{p}=0,038$ respectivamente. Las razones por las cuales los pacientes tuvieron dificultades para el acceso a la atención domiciliaria son atribuibles a que tienen sus domicilios en zonas dispersas y al aumento del servicio que superó la oferta.

Por último, se puede concluir que el resultado de las ayudas diagnósticas como causa de la estancia prolongada, se atribuye a la diversidad y complejidad de los pacientes que ingresan con estos requerimientos y que no fueron planificados para la fecha, de acuerdo al portafolio de la IPS y otras instituciones hospitalarias. Del mismo modo, se debe tener en cuenta las restricciones de recurso humano, lo que afecta la oportunidad y la pertinencia en la ejecución de las necesidades de cada paciente, durante la hospitalización y definición de su conducta.

\section{Discusión}

La estancia hospitalaria prolongada es un indicador indirecto de la calidad del cuidado otorgado a los pacientes en las unidades de segundo y de tercer nivel, ya que un procedimiento retrasado, omitido o excesivo prolongará su estancia hospitalaria, incrementando los costos (3).

Según Ceballos, Velásquez y Posada, en su estudio sobre duración de la estancia hospitalaria, una de las 


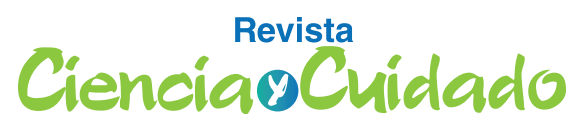

Scientific Journal of Nursing

causas más frecuentes de la estancia prolongada fue la demora en la realización de procedimientos quirúrgicos y diagnósticos con un valor de $67,6 \%$ del total de las referencias encontradas (1). De igual manera, Carey, Sheth y Braithwaite (11), encontraron que los retrasos en los servicios médicos se debieron con mayor frecuencia al aplazamiento de los procedimientos (54\%).

En este estudio, también se descubrió que entre los posibles factores que contribuyen a la estancia hospitalaria prolongada están: la atención domiciliaria $(\mathrm{p}=0,028)$; y los procedimientos: $(\mathrm{p}=0.046)$ y ayudas diagnósticas: $(p=0,38)$. Es importante resaltar, además, que, según la información suministrada por personal administrativo de la institución, este resultado se debe a que para el año 2018, esta entidad no contaba con disponibilidad de un resonador, factor que incidió en que se prolongase la hospitalización en muchos de los pacientes (12).

Se debe precisar, que la administración en salud comprende temas fundamentales como la optimización y pertinencia del uso de los recursos físicos en las organizaciones, elementos indispensables para el cumplimiento de metas y objetivos propuestos por las instituciones prestadoras de salud; es desde este punto de vista, que la estancia hospitalaria prolongada trae consecuencias desfavorables como sobreocupación de la capacidad instalada, lo que conlleva a la disminución en la recepción de nuevos pacientes. En la actualidad la estancia hospitalaria prolongada se debe entre otros factores a: un inadecuado diagnóstico o tratamiento, pacientes en estudio más allá de los tiempos prudentes, tratamientos fisioterapéuticos, rehabilitación o radioterapia, ineficiencia de los servicios de apoyo al diagnóstico, problemas de organización en el trabajo médico y/o tardanzas en el suministro de medicamentos a la sala, entre otros (11)

Dentro de las variables sociodemográficas se analizó el sexo de los pacientes registrados, con el fin de identificar si uno u otro tienen factores de riesgo que incidan en una estancia hospitalaria prolongada; al respecto, del total de registros el $49.83 \%$ (145) fueron mujeres y el $50.17 \%$ (146) hombres. Se determinó que, en la distribución porcentual por sexo, no hay una variación significativa entre ambos. Al mismo tiempo, Loren y Gascón en su investigación sobre "variables biopsicosociales relacionadas con la duración de estancia hospitalaria en personas mayores" (13) demostraron que un $53,7 \%$ eran hombres y un $46,3 \%$ mujeres, lo que indica que la estancia prolongada, en cuanto a esta variable, no es significativa, siendo por tanto una población homogénea. Solamente se encuentra diferencia significativa en cuanto a la relación del sexo, puesto
Factores de estancia hospitalaria prolongada en una

institución de salud de Medellín que el más afectado fue el sexo femenino con un $60 \%$, según lo señalan Abando y Arévalo en su investigación (9).

Con respecto a la edad se identificó que el rango donde más se concentran los pacientes esta entre 61 a 75 años con un porcentaje de 33\%. Por su parte, Loren y Gascón revelan una media de edad de 81, 24 años. Estos investigadores asocian la edad con uno de los factores más determinantes para tener estancias hospitalarias prolongadas, como es la población adulta, presente en mayor proporción. Este estudio también se concentra en la población del adulto mayor, pero con una variación en edades inferiores que para el estudio anterior. Por el contrario, Abando y Arévalo en su investigación "Características personales, clínicas y sociales en pacientes con estancia hospitalaria prolongada" encontraron que los grupos etarios más afectados fueron los jóvenes y adultos hasta en un $45 \%$, mediana 35,89 y desviación estándar (DE) de 10,45 $(9,14)$.

En relación al aspecto socioeconómico de los pacientes, el $55 \%$ pertenece a el estrato 3 . Este resultado contrasta con el estudio de Ceballos y Velásquez quienes argumentan que después de la revisión bibliografía que efectuaron, descubrieron que la duración de la estancia hospitalaria también se ve afectada por las características del paciente (1). Según estos investigadores la situación socio-familiar del paciente condiciona el apoyo a los cuidados en el domicilio; así como el rechazo por parte de la familia para aceptar el traslado a un hogar de ancianos, o las condiciones inadecuadas de las viviendas. Sin embargo, no se clasifica por estrato socioeconómico para indicar que esté relacionado con la estancia hospitalaria prolongada, pero sí se presentan las características antes mencionadas, que van de la mano con las condiciones económicas del paciente y su núcleo familiar. En este estudio se encontró una similitud en cuanto a la dificultad para la atención domiciliaria, debido a las condiciones de vivienda y georreferencia de la misma.

Conforme al estudio de Ceballos, Velásquez y Jaén (1) que hace referencia a la administración hospitalaria, señala que la larga estancia de los pacientes está asociada a la eficiencia en la interacción del servicio de hospitalización con otros servicios del hospital como son los tiempos de respuesta de los procedimientos quirúrgicos y diagnósticos. Sin embargo, no se analizaron las variables que expliquen la dinámica entre los servicios y los tiempos de respuesta. También, se observó que la especialidad con mayor estancia hospitalaria prolongada fue la no quirúrgica, distribuida entre medicina interna, neurología, neumología y cardiología, entre otras, con 


\section{Ciencià Cuidado}

Scientific Journal of Nursing

un total de $74,9 \%$ equivalente a 218 registros.

Por otro lado, autores como Fernández y Represas (15) afirman que las variables relacionadas con la esfera social juegan un papel relevante en la estancia hospitalaria, además del impacto de la enfermedad y la persistencia del tabaquismo en pacientes con enfermedad pulmonar obstructiva crónica (EPOC) grave (16).

Es necesario aclarar que en el caso de que el paciente tenga una patología respiratorita crónica, tiene como consecuencia una comorbilidad mayor o igual a 3 y un puntaje mayor o igual a 3 en el índice del grado de severidad, para las Neumonías adquiridas en la Comunidad (CURB65). Estas patologías aumentan el riesgo de permanecer por más tiempo en el hospital; y en el caso de pertenecer al sexo femenino, se considera como factor protector. La patología respiratoria crónica en el sexo femenino, CURB65 es mayor o igual a tres y comorbilidad mayor o igual a tres, lo que muestra una asociación independiente respecto a la estancia hospitalaria prolongada (17).

Según lo expresado por Valdivieso, Arévalo \& Orihuela (9), la estancia hospitalaria está afectada por factores personales como el sexo y estado civil. Agregan que también influyen la procedencia, factores clínicos como la vía y la condición de ingreso; así como lo social que tiene que ver con el acompañamiento de un familiar y de otros no emparentados. Estos elementos inciden en pacientes ingresados por motivo clínico.

En cuanto a la existencia de riesgo de desnutrición preoperatoria, se ha comprobado su relación con la estancia hospitalaria prolongada en pacientes que han sido operados por cirugía gastrointestinal. Por tanto, en estudios subsecuentes se deberá analizar todo lo relacionado con la implementación de estrategias nutricionales que incidan en que la estancia en el hospital sea de menos tiempo (18).

Se debe mencionar que son múltiples los factores que afectan la estancia prolongada en pacientes, esto conlleva a que aumenten ciertos riesgos, como la desnutrición, de acuerdo a recientes investigaciones.

Por su parte, los investigadores Ramos, Soto, Martínez, Herrera y Carrera $(19,20)$, confirman que la sarcopenia es una condición que afecta a una gran proporción de ancianos hospitalizados y se asocia a un mayor riesgo de muerte durante la hospitalización. La sarcopenia, se caracteriza por la pérdida progresiva y generalizada de la masa muscular, la fuerza y el rendimiento físico.
Natalia Cañas Benítez, Erika Moreno Pareja, Edwin Alexis Cataño Saldarriaga.

Conviene señalar que la patología respiratoria Displasia broncopulmonar (DBP) y la cardiopatía congénita, se asociaron con estancias hospitalarias prolongadas. Los pacientes menores a 6 meses fueron el grupo etario con mayor prevalencia de bronquiolitis, por consiguiente necesitan una estancia hospitalaria prolongada. Es por eso, que una de las enfermedades que se debe tener en cuenta, tiene que ver con la respiración, por su relación estrecha con la patología en estudio, constituyéndose en un problema socioeconómico directo, lo que da lugar a frecuentes citas médicas particulares y visitas a emergencias con riesgo de reingreso y estancia prolongada $(21,22)$.

Hay que señalar que el promedio de estadía es un indicador que se utiliza para poder evaluar todo lo referente a la eficiencia, en cuanto al aprovechamiento de las camas para las hospitalizaciones, así como recurso básico en este ambiente; además, de forma indirecta permite estimar la agilidad en cuanto a los servicios en los hospitales. Sobre el tiempo de estancia es adecuado o apropiado cuando se ajusta a los días necesarios de ingreso en una unidad de salud, considerándose prolongado al superar su media estandarizada. Entre los distintos continentes se documenta una variabilidad más larga en los estadounidenses $(15,23,24)$.

En realidad la estancia hospitalaria prolongada constituye una preocupación mundial, ya que genera efectos negativos en el sistema de salud como, por ejemplo: aumento en los costos, deficiente accesibilidad a los servicios de hospitalización, saturación de las urgencias y riesgos de eventos adversos $(1,25,26)$.

Según diversos autores, para un paciente con cáncer de cabeza, su estancia hospitalaria depende fundamentalmente del tiempo que tarde en recuperar la función deglutoria y poder alimentarse por la boca; $y$, en segundo lugar, está en función de las complicaciones infecciosas del postoperatorio.

Se debe precisar que ni el estado nutricional del enfermo, ni la localización del tumor inciden de forma directa en la estancia hospitalaria de estos enfermos. Igualmente, se debe tener en cuenta que los recientes avances en el equipamiento y en las técnicas quirúrgicas han mejorado el postoperatorio en este campo, permitiendo una recuperación precoz, un menor grado de dolor y tasa de infección, una menor estancia hospitalaria e incluso unos mejores resultados $(27,28)$. Varios autores expresan que los días de hospitalización, en algunos casos no son necesarios. Las principales razones que fundamentan lo dicho antes, tienen que ver con: la falta de disponibilidad de instalaciones de atención prolongada, razones del paciente o de la familia, demoras 


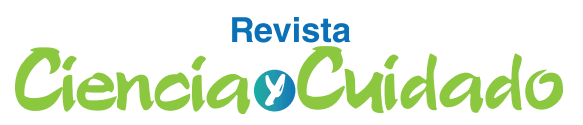

Scientific Journal of Nursing en los procedimientos y en la programación de pruebas $(29,30)$.

De acuerdo a los antecedentes encontrados, y la perspectiva de diferentes investigadores sobre la estancia hospitalaria prolongada, se evidencia que la gran problemática que hay detrás de esto, se debe a que algunas veces el número de días es innecesario y eso afecta a los familiares, sobretodo porque depende de procesos internos del hospital; no obstante, existen riesgos asociados a la estancia hospitalaria prolongada que pueden comprometer la salud del paciente, y de esa manera se congestiona el sistema.

\section{Conclusiones}

- El género, mujer u hombre, no es indicador que represente mayor preocupación o interés en este estudio, ya que la tendencia del resultado indica que no existe una prevalencia hacia ninguno de los dos sexos; por el contrario, se indica que hay cierto equilibrio en el número de mujeres y hombres que permanecen por una mayor estancia en la institución.

- El rango de edad en los pacientes supera los 60 años, indicando que la población de los pacientes es de edad adulta; lo que no indica que la estancia sea mayor si se tiene en cuenta que la mayoría de los casos se deben a ayudas diagnósticas.

- La estancia de un paciente, no solo depende de su condición clínica, sino que además existe una interrelación con otros actores en su atención, de la institución, del prestador y de la red de servicios. En este estudio se logró identificar que los factores que más se asociaron a la estancia hospitalaria prolongada en una institución prestadora de servicios de salud de mediana y alta complejidad de la ciudad de Medellín durante el año 2018, fueron: la atención domiciliaria, ayuda diagnóstica y procedimiento. Estos tres elementos estadísticamente presentaron una asociación con el fenómeno evaluado. Es importante destacar que, en el análisis realizado en este estudio en cuanto a la variable de eventos adversos, no se encontraron registros que demostraran que la estancia hospitalaria prolongada en esta institución se deba a esta causa.

- El factor económico de los pacientes hay que tenerlo en cuenta, puesto que se presentan dificultades en el regreso a sus domicilios después de la alta médica autorizada.

En cuanto a las limitaciones del estudio por ser una fuente secundaria de información, no se presentaron dificultades en el acceso a la misma, ni dilemas éticos que comprometieran la integridad de la población objeto de estudio.

\section{Conflicto de intereses}

Los autores declaran no tener ningún conflicto de intereses.

\section{Referencias Bibliográficas}

1. Ceballos-Acevedo T, Velásquez-Restrepo P, Jaén-Posada J. Duración de la estancia hospitalaria. Metodologías para su intervención. Rev Gerenc Polít Salud. 2014;13(27):274-95.

2. Aguirre GH, García MM, Garibaldi ZJ. Los factores asociados con la estancia hospitalaria prolongada en una unidad de tercer nivel. Gac méd Méx. 1997;133(2):71-7.

3. López-Pardo P, Socorro-García A, Baztán-Cortés J. Influencia de la duración de la estancia hospitalaria sobre la mortalidad tras el alta en pacientes mayores con patología médica aguda. Gaceta Sanitaria. octubre de 2016;30(5):375-8.

4. Ruiz-Gómez F, editor. Precios y contratos en salud: estudio indicativo de precios y análisis cualitativo de contratos [Internet]. Bogotá: Ministerio de la Protección Social, MPS [u.a.]; 2008. 406 p. Disponible en: https:// www.researchgate.net/publication/306611989 Precios y contratos en salud Estudio indicativo de precios y analisis cualitativo de contratos

5. Palacios-Barahona A, Bareño-Silva J. Factores asociados a eventos adversos en pacientes hospitalizados en una 
Scientific Journal of Nursing

entidad de salud en Colombia. CES Medicina. enero de 2012;26(1):19-28.

6. Mora S, Barreto C, García L. Procesos médico-administrativos en Hospitalarios y prolongación de la estancia. Hospital de San José, Bogotá D.C. Colombia. Repert Med Cir. el 1 de diciembre de 2015;24(4):284-93.

7. Fleury S. ¿Universal, Dual o Plural? Modelos y dilemas de atención de la salud en América Latina. [Internet]. Banco Interam Desarro Inst Interam Para El Desarro Soc INDES; 2002. Disponible en: https://www.researchgate.net/publication/239597859

8. Alvarado B, Rodríguez-Rojas Y, Piñeros J. Costos Financieros de la Baja Calidad Por Aumento de la Estancia Hospitalaria. Rev Glob Negocios. 2017;5(7):55-62.

9. Abanto R, Valdivieso-Jiménez G, Arévalo A, Orihuela S. Características personales, clínicas y sociales en pacientes con estancia hospitalaria prolongada en los pabellones de pacientes agudos del Hospital Víctor Larco Herrera. Horizonte Médico (Lima). abril de 2018;18(2):60-70.

10. Martínez-Ramírez A, Chávez-Ramírez S, Cambero-González E. Factores Inhibidores de la Calidad en los Servicios de Salud. Análisis desde la perspectiva de los Hospitales privados y públicos. Rev CONAMED. 2009;14(4):5-14.

11. Carey M, Sheth H, Braithwaite R. A prospective study of reasons for prolonged hospitalizations on a general medicine teaching service. J Gen Intern Med. febrero de 2005;20(2):108-15.

12. Pérez-Rubio A, Santos S, Luquero FJ, Tamames S, Cantón B, Castrodeza JJ. Evaluación de la adecuación de las estancias en un hospital de tercer nivel. Anales Sis San Navarra [Internet]. abril de 2007 [citado el 15 de abril de 2021];30(1). Disponible en: http://scielo.isciii.es/scielo.php?script=sci arttex$\underline{\text { t\&pid }=\text { S1137-66272007000100003\&lng }=\text { en\&nrm }=\text { iso\&tlng }=\text { en }}$

13. Lorén-Guerrero L, Gascón-Catalán A. Biopsychosocial factors related to the length of hospital stay in older people. Rev Latino-Am Enfermagem. diciembre de 2011;19(6):1377-84.

14. Sánchez-Hernández E, Pérez-Fouces F, López-Castillo E, de la Torre Vega G, Velez-Fernández G. Factores de riesgo asociados a estadía hospitalaria prolongada en pacientes adultos. MEDISAN. abril de 2019;23(2):271-83.

15. Fernández-García S, Represas-Represas C, Ruano-Raviña A, Botana-Rial M, Mouronte-Roibas C, Ramos-Hernández C, et al. Predictores sociales y clínicos asociados con estancia hospitalaria prolongada en pacientes con agudización grave de EPOC. Revista Clínica Española. el 1 de marzo de 2020;220(2):79-85.

16. Santamaria A, Romero-Marin M, Romero-Rondón S, Herrera-Herrera M, Robayo-Sánchez K, Bautista-Granados D. Factores asociados a estancia prolongada en una población de pacientes con exacerbaciones de EPOC en un hospital de referencia de Nororiente colombiano. Revista Americana de Medicina Respiratoria [Internet]. 2019 [citado el 15 de abril de 2021];19(4). Disponible en: http:/www.ramr.org/articulos/volumen_19 numero 4/ articulos_originales/articulos_originales factores_asociados a estancia_prolongada_en_una_poblacion_de pacientes_con_exacerbaciones_de epoc.php

17. Gonzales W. Factores asociados a estancia hospitalaria prolongada en pacientes mayores de 60 años con neumonía adquirida en la comunidad en el Hospital Militar Central del 2007 al 2016 [Internet]. [Perú]: Ricardo Palma; 2018. Disponible en: http://repositorio.urp.edu.pe/bitstream/handle/URP/1247/56\%20Fernandez\%20 Gonzales $\% 20$ Walmer.pdf?sequence $=1 \&$ isAllowed $=\mathrm{y}$

18. Peniche-Herrera J, Cortés-Telles A, Lozano-Salazar R. Riesgo de desnutrición prequirúrgico como factor causal de estancia hospitalaria prolongada en cirugía gastrointestinal. Rev Biomed. 2018;29(3):45-50.

19. Ramos-Ramirez K, Soto A, Ramos-Ramirez K, Soto A. Sarcopenia, mortalidad intrahospitalaria y estancia 


\section{Scientific Journal of Nursing}

hospitalaria prolongada en adultos mayores internados en un hospital de referencia peruano. Acta Médica Peruana. octubre de 2020;37(4):447-54.

20. Martínez-Calvache V, Herrera-Peña Á, Carrera-Gil F. Sarcopenia y fragilidad en adultos mayores hospitalizados en salas medicina interna. Acta Med Colomb. :9.

21. Guerrero M. Factores de riesgo asociados a estancia hospitalaria prolongada en pacientes con diagnóstico de bronquiolitis en el Hospital de Apoyo María Auxiliadora. 2018, enero 2016-diciembre [Internet]. [Perú]: Ricardo Palma; 2018. Disponible en: https://repositorio.urp.edu.pe/bitstream/handle/URP/1210/47MDELOSRIOS. pdf?sequence $=1 \&$ isAllowed $=\mathrm{y}$

22. Ríos-Carrión M, Gavino-Diaz G, Valverde-Balladares P, Romero-Urrea H. Perspectivas del abordaje respiratorio estratégico en post cirugía pediátrica de cardiopatía congénita. Más Vita Rev Cienc Salud. el 30 de abril de 2020;2(1):29-37.

23. Sánchez H, Pérez F, López C. Factores de riesgo asociados a estadía hospitalaria prolongada en pacientes adultos. MediSan. 2019;23(02):271-83.

24. Mayorga-Chávez D, Arnao-Noboa A, Pereira-Olmos H. Factores de Riesgo asociados con la Estancia Hospitalaria en niños con Bacteriemia por Staphylococcus Aureus: Artículo Original. REV-SEP [Internet]. el 31 de agosto de 2020 [citado el 10 de abril de 2021];21(2). Disponible en: http://rev-sepp.ec/index.php/johs/article/ view/14

25. Perales-Pardo R. A propósito de la adecuación de los ingresos hospitalarios no quirúrgicos desde un Servicio de Urgencias. Emergencias. 2004;16(3):223-8.

26. Angulo I, González G, Cedano A, Gómez BC. Relación entre el prestador de servicio de salud y la estancia prolongada en el hospital. Revista CONAMED. 2009;14(4):21-4.

27. Martín-Villares C, Domínguez-Calvo J, San Román-Carbajo J, Fernández-Pello M, Tapia-Risueño M, Pomar-Blanco P. Estancia hospitalaria prolongada en pacientes con cáncer de cabeza y cuello: el impacto del estado nutricional y los problemas faríngeos. Nutrición Hospitalaria. mayo de 2004;19(3):150-3.

28. Alcázar-Sánchez E, Bacian-Martínez S, del Toro Gil L, Gómez-Tello V. Manejo postoperatorio en UCI de cirugía de cabeza y cuello. Medicina Intensiva. el 1 de enero de 2020;44(1):46-53.

29. Kim C. Exceso de días de hospitalización en un hospital universitario: percepciones de los médicos y gestores de alta. Revista estadounidense de atención administrada. 2011;17(2):34-42.

30. Toshikazu A, Yasuharu T, Stein G, Ishimatsu S, Birrer R. Risk Factors Associated with Prolonged Hospital Stay in Admitted Patients with Psychotropic Drug Overdose. TOCCMJ. el 19 de mayo de 2008;1(1):12-6. 